\title{
cor (Pathology ( Pats) \\ CORRELATION BETWEEN UREA LEVELS IN LACRIMAL FLUID AND PATHO-PHYSIOLOGY OF DRY EYE SYNDROMES
}

\section{Dr Anil Sharma \\ Dr Narendra Kumar Keisham* \\ Dr Archana Sharma external urea supply. Urea locally formed from ocular tissues is important for the composition of the tear fluid. In eyes with dry syndromes this production is affected. Estimation of urea levels in the lacrimal fluid may prompt a newer treatment for dry eyes utilising urea. This study was done to evaluate the correlation between serum urea levels and pathophysiology of dry eye syndromes.

Materials and methods: A prospective, observational, single-centre study. 50 patients of dry eye disease were included in the subjects and 50 controls were included in the study based on schirmer's test. Tear film urea was estimated wih semi autoanalyser erba chem 5 . When sample volume was not sufficient it was diluted with sterile water and urea was estimated after calculating for dilution factor. Blood urea was estimated with Erba Chem EM 360 working on similar principle

Results: In the test group, the urea level in tear film ranged from $14.01 \mathrm{mg} \%$ to $39 \mathrm{mg} \%$ with a mean of $26.78+/-5.70 \mathrm{mg} \%$. In the controls the levels of urea in the tear film ranged from $21.06 \mathrm{mg} \%$ to $56.00 \mathrm{mg} \%$ with a mean of $41.72 \pm 6.86 \mathrm{mg} \%$.

Conclusions: Levels of urea in tear film in normal individuals without dry eyes is similar to urea levels in the blood. Tear film urea levels were significantly lower in patients with dry eye diagnosed with Schirmer's Test. Decreased tear film urea level in dry eyes is unrelated to blood urea levels and may play a role in the pathophysiology of dry eyes. This needs further investigation.

\section{KEYWORDS : Dry Eyes, Schirmer test, Serum Urea, blood urea}

\section{INTRODUCTION}

The tear film, composed of the lipid, aqueous and mucin layers, has many functions including defending the ocular surface. The tear film covering the ocular surface presents a mechanical and antimicrobial barrier and ensures an optical refractive surface ${ }^{1}$. The lipid component originates from the meibomian glands of the tarsus and forms the superficial layer of the tear film. The aqueous component contains electrolytes, water and a large variety of proteins, peptides and glycoproteins, and is primarily secreted by the lacrimal gland. Mucins are glycoproteins expressed by epithelial tissues of mucous surfaces. They protect tissues by functioning as antioxidants, providing lubrication, and inhibiting bacterial adherence. Abnormalities of the tear film, affecting the constituents or the volume, can rapidly result in serious dysfunction of the eyelids and conjunctiva and ultimately affect the transparency of the cornea.

Dry eye is a complex clinico-pathological entity involving tear film, lacrimal glands, eyelids, and a wide spectrum of ocular surface cells, including epithelial, inflammatory, immune, and goblet cells ${ }^{2}$. Dry eye conditions interfere with the ocular surface, causing corneal irregularities. It is accompanied by increased osmolarity of the tear film and inflammation of the ocular surface. The occurrence of such changes on the eye surface results in disturbances of homeostatic neurophysiologic mechanisms which further worsens the process and causes vicious pathophysiological cycles.

There is a constant effort by investigators and researchers to develop newer strategies and drugs to improve the outcomes in dry eye syndromes ${ }^{3}$. A newer area of interest is the study on the urea levels in the tear fluid. In the ocular surface there is a well- coordinated system of enzymes that can produce urea independent of external urea supply ${ }^{4}$. Urea locally formed from ocular tissues is important for the composition of the tear fluid. In eyes with dry syndromes this production is affected. Urea is used topically in dermatological practice as a moisturiser ${ }^{5}$. Estimation of urea levels in the lacrimal fluid may prompt a newer treatment for dry eyes utilising urea

\section{MATERIALS AND METHODS}

Study Design: Prospective, Observational, Single-Centre study

Study population: 50 patients of dry eye disease were included in the subject group and 50 age and sex matched controls were also included in the study.

\section{Inclusion Criteria:}

Both subjects and controls underwent schirmer's test after obtaining consent. Patients having wetting $<10 \mathrm{~mm}$ at $05 \mathrm{~min}$ were considered as having dry eyes and were enrolled in the study as subjects. Patients having wetting $>10 \mathrm{~mm}$ at $05 \mathrm{~min}$ were enrolled in the study as controls after age and sex matching.

\section{Exclusion Criteria:}

1. Patients unwilling to give consent.

2. Patients who had undergone corneal refractive surgery, cataract surgery or any other ocular surgery.

3. Patients with known kidney disease, urinary tract disease, on high protein diet and systemic diseases like heart failure, gastrointestinal bleed, severe dehydration,

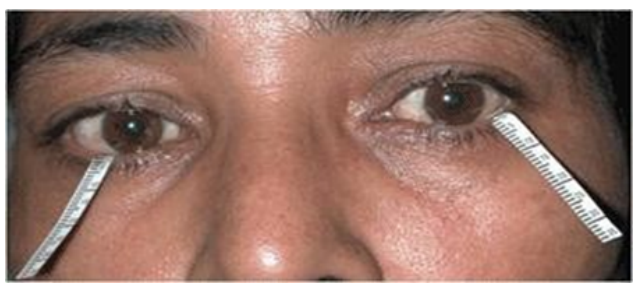

\section{Schirmer's test procedure}

Study procedure:

Schirmer's test was performed by making the subjects and controls sit in comfortable position in dimly lit room with fans and airconditioning off. Sterile Schirmer test paper (Whatman filer paper strips by Clement Clarke) manufactured by Appasamy measuring $5 \mathrm{~mm} \times 35 \mathrm{~mm}$ were used for the test. The strips were placed in the lower fornix near the lateral canthus, away from cornea and left in place for 5 minutes with eyes closed. The strip was removed after 5 minutes and the wet portion of the strip was measured in millimeters with the scale. Both eyes were tested simultaneously.

Patients having wetting $<10 \mathrm{~mm}$ at $05 \mathrm{~min}$ in both eyes were considered as having dry eyes and were enrolled in the study as subjects. Patients having wetting $>10 \mathrm{~mm}$ at $05 \mathrm{~min}$ in both eyes were enrolled in the study as controls after age and sex matching. All enrolled patients were then subjected to the assessment of urea in the tear film and simultaneously an intravenous sample was taken for estimation of urea levels in the blood. 
Tear film urea was estimated wih semi autoanalyser erba chem 5. When sample volume was not sufficient it was diluted with sterile water and urea was estimated after calculating for dilution factor.

Principle : Urease hydrolyzes urea to ammonia and $\mathrm{CO} 2$. The ammonia formed further combines with $\alpha$ Ketoglutarate and NADH to form Glutamate and NAD. The rate of oxidation of NADH to NAD is measured as a decrease in absorbance in a fixed time, which is proportional to the urea concentration in the sample. Urea $+\mathrm{H} 2 \mathrm{O}+$ $2 \mathrm{H}+$------(urease enzyme)----- 2NH4+ CO2. 2NH4 + $2 \alpha$ Ketoglutarate + 2 NADH ---------(GLDH)----

$2 \mathrm{~L}-$ glutamate $+2 \mathrm{NAD}++2 \mathrm{H} 2 \mathrm{O}$

Blood urea was estimated with Erba Chem EM 360 working on similar principle

\section{DATAANALYSIS}

The data was tabulated and subjected to statistical analysis. The software used for statistical analyses was SSPS Ver 17

\section{RESULTS AND DISCUSSION}

The age ranged from 24 yrs to 63 yrs in the subjects and from 19 yrs to 63 yrs in the controls. The mean age of the subjects was 42.83 and that of control 42.89 with no statistically significant difference between the two thereby meaning that both the groups were matched for age. The female to male ratio was 27:23 among the subjects and 24:26 in control group inferring thereby that the subjects and the controls were sex matched.

\section{Table 1.Age and Sex Description}

\begin{tabular}{|c|c|c|c|}
\hline & Dry Eye & Normal Eye & P-Value \\
\hline Age & 42.8333 & 42.8269 & 0.998 \\
\hline \multicolumn{4}{|c|}{ Age } \\
\hline \multicolumn{4}{|c|}{} \\
\hline 42.83 \\
42.83 \\
42.83 \\
42.83
\end{tabular}

Figure 1 : Average age in subjects and controls

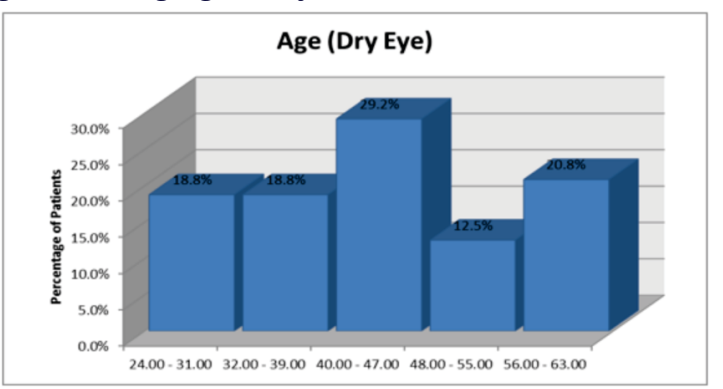

Figure 2: Age distribution in subjects

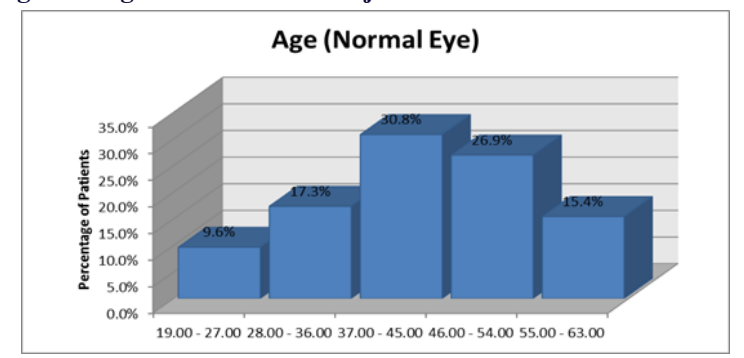

Figure 3: Age distribution in controls

In the subjects the levels of urea in the tear film ranged from 14.01 $\mathrm{mg} \%$ to $39 \mathrm{mg} \%$ with a mean of $\mathbf{2 6 . 7 8}+\mathbf{5 . 7 0} \mathbf{~ m g} \%$. In the controls the levels of urea in the tear film ranged from $26.01 \mathrm{mg} \%$ to $56.00 \mathrm{mg} \%$ with a mean of $\mathbf{4 1 . 7 2} \pm \mathbf{6 . 8 6} \mathbf{~ m g \%}$. This difference between the two mean values was statistically significant with a $\mathrm{p}$ value of 0.000 and seems to suggest that there is decreased levels of tear film urea in dry eyes when compared to normal individuals. This finding corroborates with the study of Jaeger et $\mathrm{al}^{4}$.

Table 2 : Urea levels in blood and tear film samples in subjects and controls

\begin{tabular}{|c|c|c|c|}
\hline & Dry eye & Normal eye & P-Value \\
\hline Blood Urea & $38.36 \pm 8.73$ range & $38.39 \pm 7.98$ range & .986 \\
\hline Tear Film Urea & $26.78 \pm 5.70$ range & $41.72 \pm 6.86$ range & .0001 \\
\hline
\end{tabular}

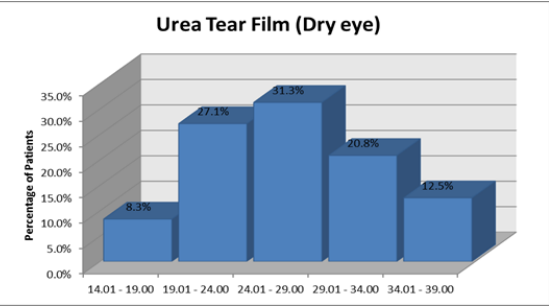

Figure 4: Urea Level in tear film in subjects

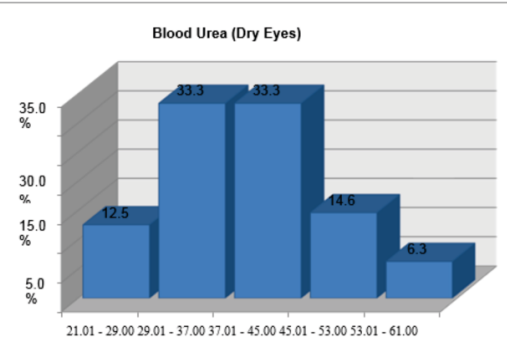

Figure 5: Urea Level in blood in subjects

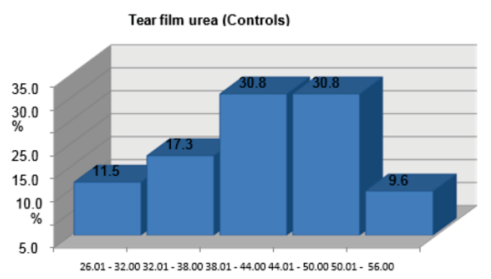

Figure 6: Urea Level in tear film in controls

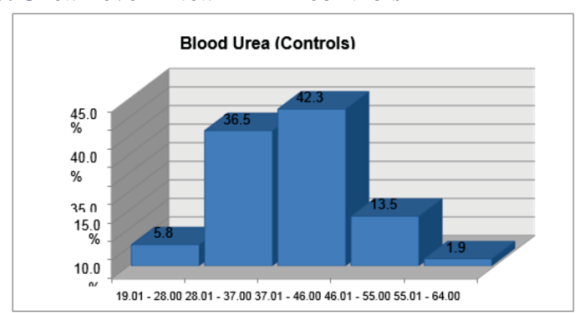

Figure 7: Urea Level in blood in control

Average Blood urea level in control population was $38.39+/-7.98$ $\mathrm{mg} \%$ with values ranging from $19.01 \mathrm{mg} \%$ to $64 \mathrm{mg} \%$. Eight values were above $46.01 \mathrm{mg} \%$.Three fourths $(78.8 \%)$ of the values were between $28.01 \mathrm{mg} \%$ to $46 \mathrm{mg} \%$. Blood urea levels in subject population was $38.36+/-8.73 \mathrm{mg} \%$ with values ranging from $21 \mathrm{mg} \%$ to $61 \mathrm{mg} \%$. Ten values ranging above $46.01 \mathrm{mg} \%$. There was no statistically significant difference in the blood urea levels in subjects and controls with a $\mathrm{p}$ value of 0.986 . This would imply that the tear fluid urea was not related to the urea levels in the blood. This was also suggested by the insignificant difference in the values of urea in the blood and tear fluid in the controls ( $38.39 \pm 7.98 \mathrm{mg} \%$ and $41.72 \pm 6.86$ $\mathrm{mg} \%$ respectively )

\section{CONCLUSIONS}

Levels of urea in tear film in normal individuals without dry eyes is similar to urea levels in the blood. Tear film urea levels were significantly lower in patients with dry eye diagnosed with Schirmer's Test. The difference in the urea levels in tear fluid in dry eyes diagnosed with shirmer's test and control group has a p value 0.0001 . There was no correlation between tear film urea compared with corresponding blood 
urea levels neither in subjects nor in controls. However the urea levels in the tear fluid of subjects with dry eyes is significantly less when compared with the control group. This finding suggests that there is a separate source of production of urea in the conjunctiva. Decreased tear fluid urea level in dry eyes is unrelated to blood urea levels and may play a role in the pathophysiology of dry eyes. This needs further investigation.

\section{Financial support and sponsorship}

Nil

\section{Conflict of interest}

\section{There are no conflicts of interest}

\section{REFERENCES:}

1. Stankiewicz A, Mikita A. Physiology and pathology of tear film in the course of dry eye syndrome. Klin Oczna. 1998; 100(5):323-9.

2. McGinnigle S, Shehzad A. Evaluation of Dry Eye. Surv Ophthalmol.2012;57 (4):29331

3. Van Haeringen NJ, Glasius E. Collection method dependent concentrations of some metabolites in human tear fluid, with special reference to glucose in hyperglycaemic

conditions. Arch Klin Exp Ophthalmol. 1977. Mar 29; $202(1): 1-7$.
4. Jager K, Kielstein H, et al. Enzymes of urea synthesis are expressed at the ocular surface, Jager K, Kielstein $\mathrm{H}$, et al. Enzymes of urea synthesis are expressed at the ocular surface,
and decreased urea in the tear fluid is associated with dry-eye syndrome. Graefes Arch and decreased urea in the tear fluid is associated
Clin Exp Ophthalmol. 2013;251(8)1995-2002.

5. Shah M K. Urea ointment (40\%). Indian J Dermatol Venereol Leprol 2003; 69:421-2. 